\title{
A study on carbapenemase producing Acinetobacter sp., and identification of OXA- 51 gene in isolates from patients attending a tertiary care hospital
}

\author{
Pradha Velu', Ramya Rengaraj, ${ }^{2, *}$ \\ Assistant Professor, Dept. of Microbiology, Adhiprasakthi Institute of Medical Sciences and Research, Tamil Nadu, India \\ *Corresponding Author: \\ Email: ramyarengaraj5@gmail.com
}

\begin{abstract}
Introduction: Acinetobacter sp., are Gram-negative non-fermenters that causes nosocomial infections. Multi drug resistance has been evolving very rapidly in Acinetobacter sp with also a dramatic increase in carbapenem resistance. Even though there are various mechanisms prevailing for such resistance, detection of Class D beta lactamases (oxacillinases) production is cumbersome. Accurate detection is done only by genotypic methods.

Aims and objectives: In order to estimate the prevalence of Acinetobacter sp., its antimicrobial susceptibility pattern, the following study was conducted in a tertiary care hospital. Organisms resistant to Carbapenems were further tested for OXA-51 gene identification by polymerase chain reaction.

Materials and Methods: All the study samples received during the study period were subjected to microbiological culture method and identification panel to isolate Acinetobacter sp., Antimicrobial susceptibility testing was done according to CLSI guidelines. Organisms that fit the criteria of multidrug resistant were further analysed for Carbapenem resistance by disc diffusion test and those isolates were subjected to polymerase chain reaction for identifying the presence of OXA-51 gene.

Results: A total of $102(4.8 \%)$ Acinetobacter sp., isolates were obtained from a total sample size of 2127 specimens during the study period. Over all, $60.78 \%$ of isolates were multidrug resistant (MDR) and $50.98 \%$ were imipenam (Carbapenem) resistant. All the imipenam resistant isolates were belonging to A.baumanii. A total of 52 isolates subjected to PCR showed positivity for OXA-51 gene.

Conclusion: This warrants the need for genotyping for all of the Acinetobacter isolates to understand the gene variations in resistance and to know whether there is a universal presence of OXA-51 gene in all of the Acinetobacter baumannii.
\end{abstract}

\section{Introduction}

Multidrug resistant Acinetobacter sp., has evolved over the past decades and it is causing an alarming rise as a nosocomial pathogen worldwide. ${ }^{1}$ Due to inappropriate guidelines and usage of Carbapenems widely, Carbapenem resistance is threatening all the organisms including Acinetobacter sp., Multidrug resistance is defined as Acinetobacter resistant to at least three classes of drugs: Cephalosporins, Fluoroquinolones and Aminoglycosides. ${ }^{2}$ Mechanism of such resistance is through various process, but the most important is by production of enzyme called Carbapenemases that causes hydrolysis of Carbapenems namely Imipenam, Meropenam and Ertapenam. ${ }^{3}$ Though Carbapenemases have several subclasses OXA, IMP, VIM are the most commonly encountered in non-fermenters. ${ }^{4}$ OXA (Oxacillin hydrolyzing beta lactamases) are plasmid encoded and there are several clusters in which multiple subgroups of OXA are encountered. ${ }^{5}$ Multiple evidences suggest that there is an intrinsic carbapenemase gene located in Acinetobacter sp., ${ }^{6}$ First of its kind OXA -51, a carbapenemase gene was identified in Argentina and after that several closely related variants of this gene were also identified. ${ }^{6,7}$ In order to estimate the prevalence of Acinetobacter sp., its antimicrobial susceptibility pattern, the following study was conducted in a tertiary care hospital. Organisms resistant to Carbapenems were further tested for OXA51 gene identification by polymerase chain reaction.

\section{Materials and Methods}

This cross sectional study was conducted in the Department of Microbiology over a period of two years in a tertiary care teaching hospital. Samples including pus, urine, blood, wound swab, fluids and sputum were included in the study. Exclusion criteria included were samples from patients who are already on antimicrobials and same samples that are taken repeatedly. All the samples were subjected to Gram staining as preliminary examination to look for short, Gram negative cocco-bacilli which suggests possibility of Acinetobacter sp., The samples were inoculated in Nutrient agar, Blood agar and Mac Conkey agar and incubated at $37 \mathrm{deg} \mathrm{C}$ for $24-48$ hours. Colonies showing cultural characteristics suggestive of Acinetobacter sp., were further subjected to biochemical reactions - catalase, oxidase, citrate, urease, methyl red (MR), Voges-Proskauer (VP), indole, triple sugar iron agar (TSI) and sugar fermentation tests. Special tests like growth at $42^{\circ} \mathrm{C}$ and at $44^{\circ} \mathrm{C}$, test for breakdown of carbohydrate (Oxidative Fermentative test with $10 \%$ OF lactose) were done for speciation of Acinetobacter. Pinkish colonies in MacConkey agar, acid in 10\% lactose but not in $1 \%$ lactose, acid without gas in glucose, arabinose, and xylose with growth at $44 \mathrm{degC}$ are used as identification features of Acinetobacter baumannii. Antimicrobial susceptibility testing of Acinetobacter sp., was done by Kirby-Bauer disc diffusion technique on Mueller 
Hinton agar. Following antimicrobial agents were used: Ampicillin (Amp), Amoxycillin - Clavulanic acid (Ac), Aztreonam (Az), Gentamicin (G), Ciprofloxacin (Cip), Cefpodoxime (Cep), Cefotaxim (Ctx), Ceftazidime (Caz), Ceftriaxone (Cef), Co-Trimoxazole (Co), Imipenem (I) and Piperacillin-tazobactam (Pt). Organisms that fit the criteria of multidrug resistant were further analysed for Carbapenem resistance by disc diffusion test and those isolates were subjected to polymerase chain reaction for identifying the presence of OXA-51 gene.

Polymerase chain reaction: A single colony of Acinetobacter strain was inoculated into $1-5 \mathrm{ml}$ of Luria-Bertani (LB) broth and incubated for 12-16 hrs. $1.5 \mathrm{ml}$ of broth was taken into micro centrifuge tube, centrifuge was done at $8000 \mathrm{rpm}$ for 2 minutes. The supernatant was decanted. The pelleted cells were resuspended in $250 \mu 1$ of suspension buffer (RNase solution and suspension buffer), $250 \mu 1$ of lysis buffer was added and mixed 4 to 6 times. $350 \mu \mathrm{l}$ of binding buffer was added and mixed 4 to 6 times and centrifuged for $10 \mathrm{~min}$ at $13,000 \mathrm{rpm}$.

The supernatant was then transferred to spin column, centrifuged for one minute and the flow through was discarded. 500 $\mu 1$ of wash buffer I was added to the spin column and centrifuged for 30 to 60 seconds and the flow through was discarded. $750 \mu \mathrm{l}$ of wash buffer II was added, centrifuged for 30 to 60 seconds, the flow through was discarded and this step was repeated once more. Flow through was discarded and centrifuged for one minute to remove ethanol. $50 \mu 1$ of elution buffer was added to the spin column and the sample was transferred to a new micro centrifuge tube.
Extracted plasmids were treated with $2 \mathrm{x}$ master mix, primers bal OXA51 (160bp) - F-5 'CTTGCTCGTTCGACCGAG3' R-5 'CCCATCCAGTTAACCAGCCTAC 3' and nuclease free water. PCR amplification done with thermal conditions and the product was visualized with agar gel electrophoresis; 100bp ladder DNA is used as reference. Product size corresponds to 160bp.

\section{Results}

A total of $102(4.8 \%)$ Acinetobacter sp., isolates were obtained from a total sample size of 2127 specimens during the study period. Majority of the isolates were obtained from pus $(47.1 \%)$ followed by urine $(23.5 \%)$, blood $(15.7 \%)$, sputum $(7.8 \%)$ and others (5.9\%). Among the 102 isolates, 98 (96.07\%) isolates were A.baumanii and $4(3.92 \%)$ isolates were A.lowffii.

Antimicrobial susceptibility testing (Table 1) has identified higher rates of resistance was seen for third generation cephalosporins and for penicillin group of antimicrobials. Aminoglycosides showed a lesser degree when compared to the above classes of drugs with $61.76 \%$ resistance for Amikacin and $74.6 \%$ of resistance for Gentamicin. Over all, $60.78 \%$ of isolates were multidrug resistant (MDR) and $50.98 \%$ were imipenam (Carbapenem) resistant.

All the imipenam resistant isolates were belonging to A.baumanii. None of the Acinetobacter lowffii showed imipenam resistance. A total of 52 isolates subjected to PCR showed positivity for OXA-51 gene. (Fig. 1)

Table 1: Antimicrobial susceptibility profile of study isolates $(n=102)$

\begin{tabular}{|l|c|c|c|c|}
\hline Drug & No. of sensitivity & Percentage & No. of resistance & Percentage \\
\hline Ampicillin & 3 & $2.94 \%$ & 99 & $97.06 \%$ \\
\hline Amoxy-Clav & 4 & $3.92 \%$ & 98 & $96.08 \%$ \\
\hline Amikacin & 39 & $38.23 \%$ & 63 & $61.76 \%$ \\
\hline Cefuroxime & 10 & $9.80 \%$ & 92 & $90.2 \%$ \\
\hline Ceftriaxone & 18 & $17.65 \%$ & 84 & $82.35 \%$ \\
\hline Ciprofloxacin & 40 & $39.21 \%$ & 62 & $60.78 \%$ \\
\hline Gentamycin & 26 & $25.49 \%$ & 76 & $74.6 \%$ \\
\hline Imipenam & 50 & $49.02 \%$ & 52 & $50.98 \%$ \\
\hline Meropenam & 47 & $46.08 \%$ & 55 & $53.92 \%$ \\
\hline Nitrofurantoin & 3 & $12.5 \%$ & 21 & $87.5 \%$ \\
\hline Norfloxacin & 15 & $62.5 \%$ & 9 & $37.5 \%$ \\
\hline Tetracycline & 15 & $14.70 \%$ & 87 & $85.3 \%$ \\
\hline
\end{tabular}

* Samples tested for Nitrofurantoin and Norfloxacin are (urine) $\mathrm{N}=24$ 
Fig. 1: Samples showing positivity for OXA-51 gene (160bp) in agarose gel electrophoresis

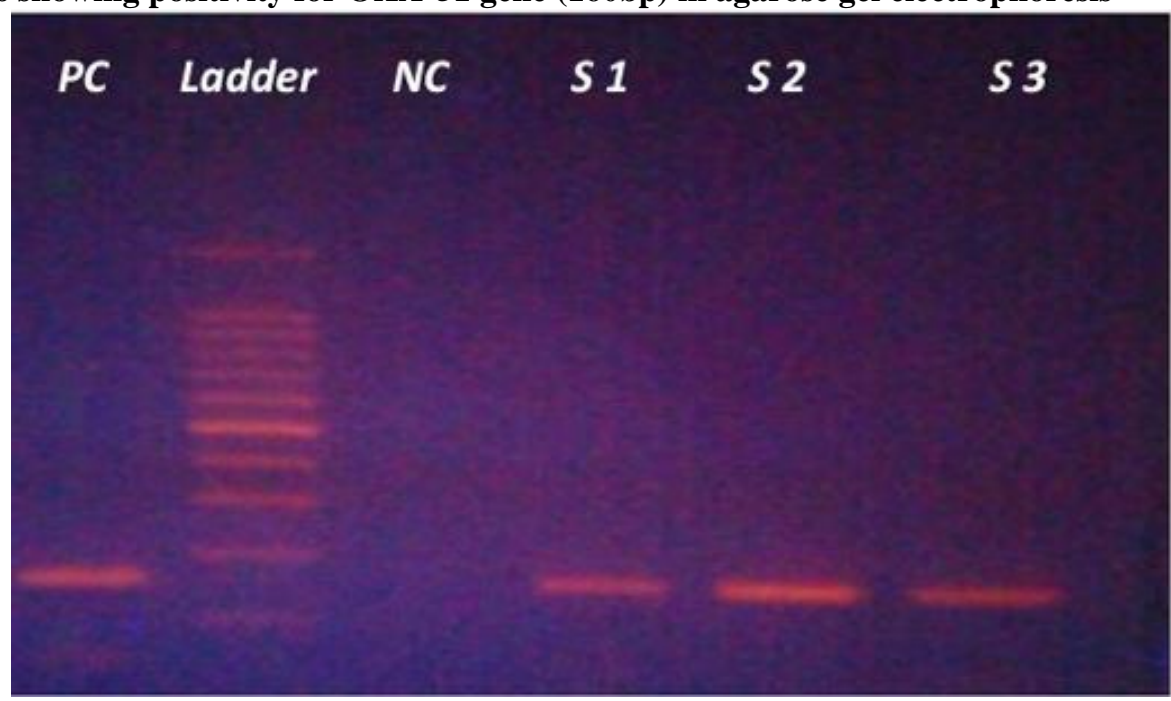

\section{Discussion}

Acinetobacter sp., are Gram-negative nonfermenters family which is nowadays most commonly encountered as nosocomial pathogen everywhere. It has an intrinsic ability to survive in adverse environmental situations and also has high level of antimicrobial resistance too. ${ }^{2}$

The above study was conducted to estimate the prevalence of Acinetobacter sp and its antimicrobial pattern with special reference to detection of OXA-51 gene. Prevalence of Acinetobacter sp was $4.8 \%$ in our hospital which is similar to another study conducted in India which showed $7.87 \% .{ }^{8}$

Speciation done among the genus have shown 98 (96.07\%) isolates were A.baumanii and 4 (3.92\%) isolates were A.lowffii. This has been proven in many studies that among all the species, Acinetobacter baumannii is the most common one. ${ }^{9}$

Antimicrobial susceptibility profile has estimated that over all, $60.78 \%$ of isolates were multidrug resistant (MDR) and 50.98\% were imipenam (Carbapenem) resistant. All the imipenam resistant isolates were belonging to A.baumanii. Drug susceptibility has shown that these isolates are becoming more resistant to most of the drugs and becoming multi drug resistant, as suggested by other study. ${ }^{10}$ In a study conducted to estimate the drug resistant prevalence, $<50 \%$ Carbapenem resistance was seen in Acinetobacter baumannii in the year before 2012 which has drastically increased to $67-74 \%$ during the year 2009-2013. ${ }^{11}$

When Carbapenems have become the last reserve for treatment, organism in turn undergoes evolution with few mechanisms like decrease in outer membrane permeability, efflux pump mechanism and production of beta lactamases.(12) Currently available methods to detect the resistance differ in their techniques and interpretation. Most of the phenotypic methods do not detect OXA type of resistance. ${ }^{13}$
Among beta lactamases, Class D type is named as oxacillinases and they are abbreviated as OXA. There are various subgroups among them namely OXA-23 (first identified), OXA-27, OXA-49, OXA-24, OXA-51 and many more. OXA-51 is unique in that it may be intrinsically seen in strains of Acinetobacter baumannii. $^{5}$

In our study, we have tested all the Carbapenem resistant isolates $(n=52)$ to look for the presence of OXA-51 gene and it is proven that $100 \%$ of the tested Acinetobacter baumannii showed bands in OXA -51. Studies have described that OXA-51 like genes are present in almost all of the strains of Acinetobacter baumannii, which means this gene detection can be used as method of identifying the organism through amplified rRNA gene restriction analysis. ${ }^{6}$

Hence forth, from the above findings it is interpreted that OXA - 51 is present ubiquitously in almost all of the Acinetobacter baumannii isolates.

\section{Conclusion}

Due to difficulties in identification of oxacillinases by phenotypic methods, genotypic method is necessary to rule our Carbapenem resistance in Acinetobacter sp., Study has proven that there is a presence of OXA -51 gene in almost all of the Acinetobacter baumannii isolates suggests it can be used as an identification method for those species along with the drug resistant OXA genes. Further more analysis need to be done by amplified rRNA gene restriction analysis to understand the predictability and accuracy of OXA-51 detection.

\section{References}

1. Pourhajibagher M, Hashemi FB, Pourakbari B, Aziemzadeh M, Bahador A. Antimicrobial Resistance of Acinetobacter baumannii to Imipenem in Iran: A Systematic Review and Meta-Analysis. Open Microbiol J. 2016;10:32-42.

2. Manchanda V, Sanchaita S, Singh N. Multidrug Resistant Acinetobacter. J Glob Infect Dis. 2010;2(3):291-304. 
3. Opazo A, Domínguez M, Bello H, Amyes SGB, González-Rocha G. OXA-type carbapenemases in Acinetobacter baumannii in South America. J Infect Dev Ctries. 2012 Apr 13;6(4):311-6.

4. Queenan AM, Bush K. Carbapenemases: the Versatile $\beta$ Lactamases. Clin Microbiol Rev. 2007 Jul;20(3):440-58.

5. Walther-Rasmussen J, Høiby N. OXA-type carbapenemases. J Antimicrob Chemother. 2006 Mar;57(3):373-83.

6. Turton JF, Woodford N, Glover J, Yarde S, Kaufmann ME, Pitt TL. Identification of Acinetobacter baumannii by Detection of the blaOXA-51-like Carbapenemase Gene Intrinsic to This Species. J Clin Microbiol. 2006 Aug 1;44(8):2974-6.

7. Brown S, Young HK, Amyes SGB. Characterisation of OXA-51, a novel class D carbapenemase found in genetically unrelated clinical strains of Acinetobacter baumannii from Argentina. Clin Microbiol Infect Off Publ Eur Soc Clin Microbiol Infect Dis. 2005 Jan;11(1):15-23.

8. Grewal US, Bakshi R, Walia G, Shah PR. Antibiotic susceptibility profiles of non-fermenting gram-negative Bacilli at a Tertiary Care Hospital in Patiala, India. Niger Postgrad Med J. 2017 Jun;24(2):121-5.

9. Nowak P, Paluchowska P. Acinetobacter baumannii: biology and drug resistance - role of carbapenemases. Folia Histochem Cytobiol. 2016;54(2):61-74.

10. Gonzalez-Villoria AM, Valverde-Garduno V. AntibioticResistant Acinetobacter baumannii Increasing Success Remains a Challenge as a Nosocomial Pathogen. J Pathog. 2016;2016:7318075.

11. Hsu L-Y, Apisarnthanarak A, Khan E, Suwantarat N, Ghafur A, Tambyah PA. Carbapenem-Resistant Acinetobacter baumannii and Enterobacteriaceae in South and Southeast Asia. Clin Microbiol Rev. 2017 Jan;30(1):1-22.

12. Migliavacca R, Espinal P, Principe L, Drago M, Fugazza $\mathrm{G}$, Roca I, et al. Characterization of resistance mechanisms and genetic relatedness of carbapenemresistant Acinetobacter baumannii isolated from blood, Italy. Diagn Microbiol Infect Dis. 2013 Feb;75(2):180-6.

13. Vijayakumar S, Gopi R, Gunasekaran P, Bharathy M, Walia K, Anandan S, et al. Molecular Characterization of Invasive Carbapenem-Resistant Acinetobacter baumannii from a Tertiary Care Hospital in South India. Infect Dis Ther. 2016 Sep;5(3):379-87.

How to cite this article: Velu P, Ramya Rengaraj R. A study on carbapenemase producing Acinetobacter sp., and identification of OXA-51 gene in isolates from patients attending a tertiary care hospital. Indian J Microbiol Res 2018;5(1):96-99. 